\title{
Calculations and measurements of harmonic current distributions in the catenary of railways with single-phase A.C.
}

\author{
A. Zynovchenko ${ }^{1}$, J. Xie $^{1}$, S. Jank $^{2}$ \& F. Klier ${ }^{2}$ \\ ${ }^{I}$ Department of Energy Conversion and Storage, University of Ulm, \\ Germany \\ ${ }^{2}$ Department of Electromagnetic Compatibility, DB Systemtechnik, \\ Munich, Germany
}

\begin{abstract}
Frequency converters find wide application in rail vehicles and power supply stations of railway networks. An important disadvantage of frequency converters is the emission of voltage and current harmonics. They cause extra stress to railway equipment and can interfere with sensitive signalling circuits resulting in their mis-operation. Therefore, propagation of current harmonics in the catenary network and resonance phenomena have to be investigated, which requires network calculations for high frequencies. A new catenary network calculation method covers the frequency range up to $20 \mathrm{kHz}$. Experimental proof of the calculation method was done. Comparison of calculations and experimental results is given.

Keywords: network model, network calculation, catenary, track circuits, frequency converter, harmonics, electromagnetic compatibility.
\end{abstract}

\section{Introduction}

The development of high power semiconductors today results in the increasing use of frequency converters in railway networks. Nowadays, most rail vehicles and also some power stations are equipped with frequency converters. Such converters generate an AC-voltage by building it with pulse shaped voltages (pulse width modulation). Because of that, the resulting output voltage of the 
converter consists of a main frequency voltage and harmonics (e.g. EtxeberriaOtadui et al. [1]).

Figure 1 shows a simple traction network. The frequency converter of a locomotive generates voltage harmonics $U_{v}^{(\text {conv) }}$. Due to the network impedance, voltage harmonics cause current harmonics. The $v$-th current harmonic $I_{v}^{(c o n v)}$ of the converter can be calculated as

$$
I_{v}^{(c o n v)}=U_{v}^{(c o n v)} / Z_{v}
$$

with $Z_{v}$ as input impedance of the network for the $v$-th harmonic seen from the converter. Current harmonics of the converter propagate into the overhead catenary. Flowing back through the rails to the converter, current harmonics can affect track circuits.

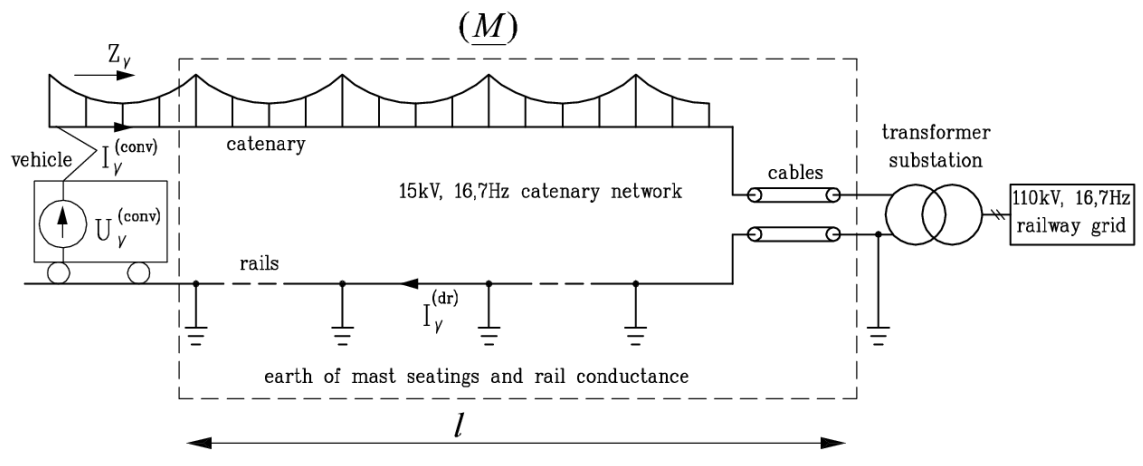

Figure 1: $\quad$ Traction network.

Track circuits are used for signalling purposes and are one of the most important parts of a railway system. They serve to detect the presence of trains on a particular track section. Track circuits of German railways operate at frequencies of 42 and $100 \mathrm{~Hz}$ as well as at audio frequencies from $4,75 \mathrm{kHz}$ up to $16,8 \mathrm{kHz}$. A track circuit uses rails as part of a circuit.

Returning back to the frequency converter, current harmonics flow also through the rails. Therefore, if a harmonic current is in the same frequency range as the operating frequency of the track circuit, it can interfere the track circuit and lead to a mis-operation of the detection relay [2].

Propagating along the catenary, current harmonics of the converter can be, depending on the network conditions, either damped or amplified. Because considered track circuits are distant from the converter place, the propagation of current harmonics along the catenary network must be investigated. The current $I_{v}^{(d r)}$ at the location of the detection relay can be calculated using a locationdependent damping ratio $D_{v}[3]$ 


$$
I_{v}^{(d r)}=I_{v}^{(\text {conv })} \cdot D_{v}=\frac{U_{v}^{(\text {conv })}}{Z_{v}} \cdot D_{v} .
$$

Magnitudes of voltage harmonics $U_{v}^{(\text {conv })}$ depend on a frequency converter type and the height of mains voltage. They can be calculated or measured for a given converter type. Thus, the influence of the converter harmonics on the track circuits is determined mainly by the damping ratio and impedance which are heavily dependent on the harmonic's frequency and network parameters such as catenary length, length of feeding cables in substations, type of catenary, location of vehicles, substations and track circuits and so on [4].

Thus, it is very difficult to make a general statement about the electromagnetic compatibility of a certain converter type basing on a few measurements. Because of that we propose to check interference limits using the model shown in figure 2. The model consists of a network model and a track circuit model. This paper is focused on the network model. Benefits and limits of known network models are discussed. Then, a new network model developed by the authors is presented. A comparative analysis of the discussed models is given. The theoretical model has been verified with the help of measurements. The results are given in the paper.

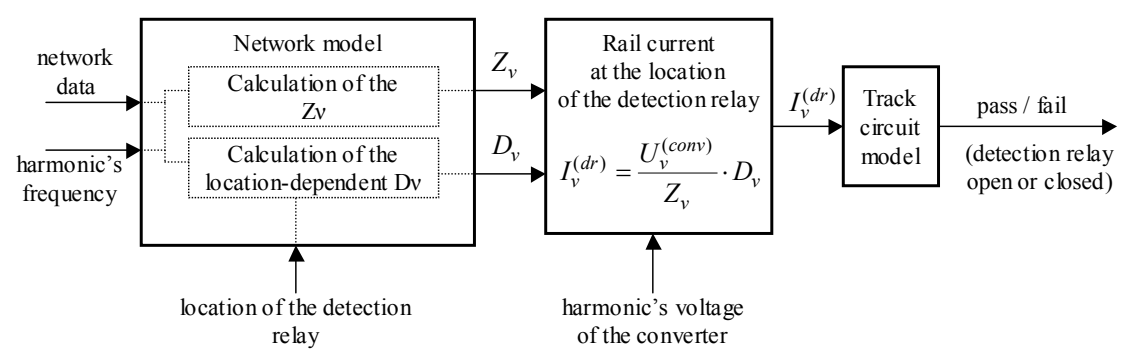

Figure 2: Model for checking interference limits.

\section{Overview of known network models}

\subsection{General requirements to a network model}

In order to check electromagnetic compatibility of frequency converters and track circuits with the help of the model figure 2, the network model must be applicable for long non-homogeneous catenary networks with railway stations, substations, tunnels and so on. A catenary can consist of many wires and be configured in any manner. The network model must be applicable for high frequencies and be able to calculate rail currents and their propagation along the track. Below, an overview of the known network models is given. 


\subsection{Network simulation model}

This approach consists in building the model of the network circuit in a simulation program such as Simulink of the MathWorks Company or Simplorer of the Ansoft Corporation. All nodes of the original network circuit must be replicated in the simulation model. In railway catenary networks with return current conduction through the soil, the earth points of catenary masts are to be considered as nodes. Because masts are usually installed every 40-80 m along the track, the number of nodes to be replicated in the simulation model is very high. For long tracks with many nodes the simulation model becomes so complex and requires so much computation costs, that it becomes no more applicable. But a simplified replication of the network in the simulation model would result in methodical errors.

\subsection{Two-wire transmission line model}

This network model is used for calculation of two-wire systems. The interrelation of input and output voltages and currents $U_{\text {in }}, I_{\text {in }}, U_{\text {out }}, I_{\text {out }}$ of a twowire system of the length $l$ is described with the well-known transmission line equations

$$
\left\{\begin{array}{l}
U_{\text {in }}=U_{\text {out }} \cdot \operatorname{ch}(\gamma \cdot l)+I_{\text {out }} \cdot Z_{w} \cdot \operatorname{sh}(\gamma \cdot l) \\
I_{\text {in }}=U_{\text {out }} \cdot \operatorname{sh}(\gamma \cdot l) / Z_{w}+I_{\text {out }} \cdot \operatorname{ch}(\gamma \cdot l)
\end{array},\right.
$$

with $\gamma$ and $Z_{w}$ as propagation factor and wave impedance of the two-wire system respectively.

Railway catenary networks, in which the soil is used as a return current conductor, must be considered as many-wire systems consisting of at least three conductors (contact line, rail and soil). In order to apply the line equations, the number of conductors must be reduced to two. For this purpose, several feeding current conductors (contact and carrying wires) and return current conductors (soil, rails and return current wires) must be consolidated [5]. The two-wire system, obtained in that way, can be calculated with the line equations (3).

However, a consolidation of wires without methodical errors is possible only if a current distribution in single wires is balanced and remains constant along the railway track. This requirement is fulfilled only for homogeneous tracks. In the vicinity of track boundaries and non-homogeneous transition areas (substations, tunnels, railway stations, rail vehicles and so on) a current distribution in rails and soil is not balanced [6]. In this case, the consolidation of conductors leads to methodical errors. Thus, the model does not meet the abovementioned requirements.

\subsection{Network model with multipoles}

The main idea of this calculation method is to represent the track with a multipole described with its transmission matrix $(\underline{M)}$ ) as shown in figure 1. In this case, the following matrix equation takes place 


$$
\left(\underline{U I}_{\text {out }}\right)=(\underline{M}) \times\left(\underline{U I}_{\text {in }}\right) .
$$

$\left(\underline{U I}_{\text {in }}\right)$ and $\left(\underline{U I}_{\text {out }}\right)$ are column vectors of the input and output voltages and currents of the multipole. The elements of the matrix $(\underline{M})$ can be calculated as described in [6] and [7]. Additionally, capacitive couplings of conductors must be considered for high frequencies.

The matrix equation (4) can be solved together with boundary conditions, which are defined by node equations for voltages and currents on the ends of the track. Currents and voltages of all conductors along the track can be computed with the help of one of the classical circuit calculation methods.

The described multipolar calculation method was implemented in a MATLAB program. Our investigations have shown, that a numeric network computation with this method is possible only for low frequencies and short-length tracks. The results computed for $16,8 \mathrm{kHz}$ were no more plausible for a track longer then approximately $5 \mathrm{~km}$. The reason for errors is the limited accuracy of representation of numeric data in the computer.

In general, the sensitivity of the solution of a system of linear equations to errors in the data can be estimated with the help of the reciprocal condition number $R C O N D$ of the matrix $(M)$. The reciprocal condition number can be computed for example with a MATLAB-Function $\operatorname{RCOND}(M)$ and can take on values between 0 and 1 . When $R \operatorname{COND}(M)$ is near 1 , the matrix is said to be well-conditioned. For a well-conditioned matrix small changes in the data always result in small changes in the solution. When $\operatorname{RCOND}(M)$ is near 0 , the matrix is called ill-conditioned. In this case small changes in the data (for example due to the limited accuracy of their numeric representation in the computer) can lead to very large changes in the solution. Thus, the solution of the system with the ill-conditioned matrix is not accurate. Our investigations for catenary networks have shown, that the reciprocal condition number tends to zero with growing track length as well as with the frequency. Thus, the matrix $(M)$ becomes ill-conditioned beginning from the certain track length. This results in big errors of the numerical solution of eqn (4). Therefore, this calculation method is limited to short-length tracks and low frequencies.

\subsection{Summary of the overview}

The overview of the known network models and their calculation methods has shown, that some models (for example, two-wire transmission line model) reproduce the real network with essential simplifications resulting in methodical errors. Other models, in which simplifications like consolidation of conductors are not mandatory, require too much computation costs (network simulations) or they cannot be calculated for long tracks and high frequencies due to numeric errors (multipolar calculation method [7]).

Thus, there is currently no model with an appropriate calculation method, which meets the requirements in full measure. 


\section{New multipolar calculation method}

The patented method for calculation of the multipolar network model has been developed by the authors. The main idea of the new method is to reduce the numeric errors of the known multipolar calculation method by improving the reciprocal condition number of the transmission matrix of the network. The improvement of this number is achieved by increasing the matrix order. The developed multipolar calculation method enables computations for long tracks and covers the frequency range up to $20 \mathrm{kHz}$.

Consider the case when the matrix $(\underline{M})$ is ill-conditioned, i.e. $\operatorname{RCOND}(\underline{M}) \approx 0$ so that eqn (4) cannot be solved directly due to numeric errors. Thus, the common multipolar method is not applicable.

In the proposed calculation method the whole track of the length $l$ is subdivided into $n$ sections of the lengths $l_{1}, l_{2}, \ldots, l_{\mathrm{n}}$ (figure 3 ). Each section can include multiple spans, masts, vehicles and so on and is represented as a multipole with its square transmission matrix $\left(\underline{M}_{i}\right), i=1 . . n$. For voltages and currents at the beginning of the track are related to those at the end of the track by the equation

$$
\left(\underline{U I}_{\text {out }}\right)=\left(\underline{M}_{n}\right) \times \ldots \times\left(\underline{M}_{l}\right) \times\left(\underline{U I}_{\text {in }}\right)=(\underline{M}) \times\left(\underline{U I}_{\text {in }}\right) .
$$

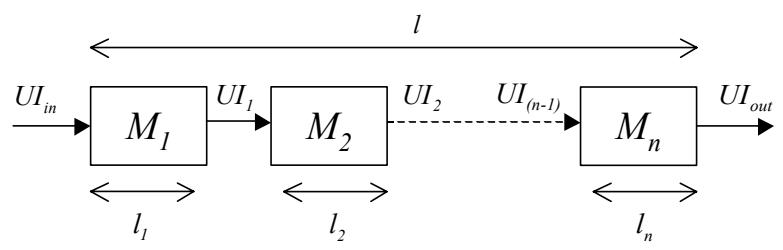

Figure 3: Subdivision of the track in sections.

The following matrix equations take place for boundaries of each section

$$
\left\{\begin{array}{l}
0=\left(\underline{M}_{1}\right) \times\left(\underline{U I}_{\text {in }}\right)-\left(\underline{U I}_{2}\right) \\
\vdots \\
0=\left(\underline{M}_{n}\right) \times\left(\underline{U I}_{n-1}\right)-\left(\underline{U I}_{\text {out }}\right)
\end{array},\right.
$$

with $\left(\underline{U I}_{1}\right) \ldots\left(\underline{U I}_{n-1}\right)$ as matrices of input and output voltages and currents of the corresponding sections.

The subdivision in sections is to be made in such a way, that the reciprocal condition number of the matrix of each section is big enough to solve each of the equations (6) with a given accuracy [8]. form

Boundary conditions on the ends of the track can be expressed in a matrix 


$$
\left(\underline{B}_{\text {in }}\right)=\left(\underline{A}_{\text {in }}\right) \times\left(\underline{U I}_{\text {in }}\right), \quad\left(\underline{B}_{\text {out }}\right)=\left(\underline{A}_{\text {out }}\right) \times\left(\underline{U I}_{\text {out }}\right),
$$

with $\left(\underline{B}_{\text {in }}\right),\left(\underline{B}_{\text {out }}\right)$ as column vectors and $\left(\underline{A}_{\text {in }}\right),\left(\underline{A}_{\text {out }}\right)$ as matrices of the boundary conditions.

The eqns (6) and (7) can be consolidated together into a single matrix equation

$$
\left(\begin{array}{c}
0 \\
0 \\
\ldots \\
0 \\
\underline{B}_{\text {in }} \\
\underline{B}_{\text {out }}
\end{array}\right)=\left(\begin{array}{ccccc}
\underline{M}_{1} & -I & \ldots & 0 & 0 \\
0 & \underline{M}_{2} & \ldots & 0 & 0 \\
\ldots & \ldots & \ldots & \ldots & \ldots \\
0 & 0 & \ldots & \underline{M}_{n} & -I \\
\underline{A}_{\text {in }} & 0 & \ldots & 0 & 0 \\
0 & 0 & \ldots & 0 & \underline{A}_{\text {out }}
\end{array}\right) \times\left(\begin{array}{c}
\underline{U I}_{\text {in }} \\
\underline{U I}_{1} \\
\underline{U I}_{2} \\
\ldots \\
\underline{U I}_{\text {out }}
\end{array}\right),
$$

with $(I)$ as identity matrix of the same order as $\left(\underline{M}_{1}\right) \ldots\left(\underline{M}_{n}\right)$.

With the help of matrix theory [9] it can be shown, that the matrix in eqn (8) is better conditioned than the matrix $(\underline{M})$ in eqn (4). Thus, errors of the numeric solution of eqn (8) are also smaller. They can be kept within desired tolerance limits by defining the total number of sections and the length of the each section.

The accuracy of the numeric solution increases with growing number of subdivisions and reducing lengths of the sections. But the order of the matrix in eqn (8) and therefore computation costs grow as well. Thus, the subdivision should be performed in such a way that on the one hand the number of sections is as low as possible. On the other hand, the solution of eqns (6) must be accurate enough for each section, i.e. the reciprocal condition number of the each matrix $\left(\underline{M}_{i}\right)$ must be big enough. In order to achieve the optimal subdivision of the track, a special iterative algorithm described in [8] should be used.

\section{Comparison of calculation methods}

In order to compare different network calculation methods, they have been applied to the double-track network (figure 1). A 1,3-km long four-track railway station, which is ca. $8 \mathrm{~km}$ distant from the frequency converter, was considered.

Figure 4 shows the rail current calculated for $16,8-\mathrm{kHz}$ harmonic with three methods. The $16,8-\mathrm{kHz}$ harmonic voltage of the converter was taken $U_{16,8 \mathrm{kHz}}^{(\text {conv }}=1 \mathrm{~V}$. One can see, that the results form the common multipolar method become unfeasible from $5,5 \mathrm{~km}$ due to numeric errors. In contrast, the new multipolar calculation method provides plausible results along the whole track. The results obtained with the two-wire transmission line model diverge from those of the new multipolar method. This is to lead back to the methodical errors due to a non-balanced current distribution between rails and soil in the near of railway station and track ends. 


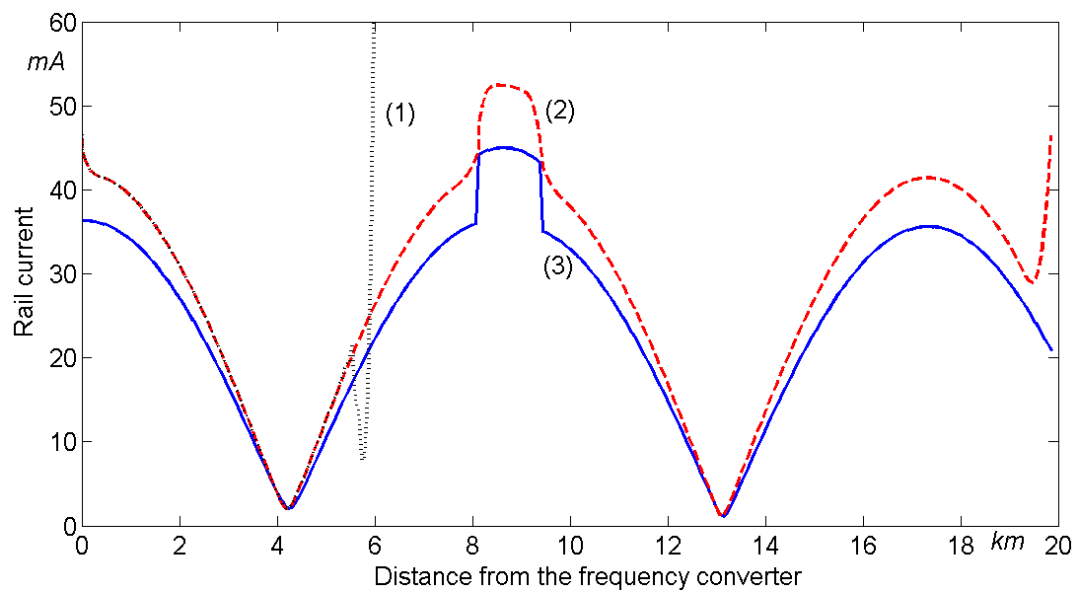

Figure 4: Rail current of the $16,8-\mathrm{kHz}$ harmonic, calculated with the common multipolar method (1), new multipolar method (2), and the two-wire transmission line method (3).

\section{Comparison of calculation and measurement results}

In order to check the correctness of the new calculation method, measurements were done on a $25 \mathrm{~km}$ long single track suburban line consisting of two rails of the type S54, a carrying wire of the type Ri100 and a traction wire of the type Bz50. The supply voltage of $15 \mathrm{kV}$ of the overhead catenary was powered off during the measurements. By means of a wave generator and a power amplifier the one end of the catenary was fed with a sine voltage $U_{\text {in }}=200 \mathrm{~V}$ with frequencies from $16,7 \mathrm{~Hz}$ up to $18 \mathrm{kHz}$. The other end of the catenary was shortcircuited to the rail through the measuring resistor of $1 \Omega$. The feeding current $I_{\text {in }}$ and the current $I_{K}$ over the measuring resistor at the short-circuit place were measured. With the measured values the track impedance $Z=U_{\text {in }} / I_{\text {in }}$ and the damping ratio $D_{K}=I_{K} / I_{\text {in }}$ were computed.

Before doing measurements, the frequency-dependent impedance and the damping ratio have been calculated with the help of the network model. Measure and calculation results are shown in figure 5 (curves 1 and 2).

The similar character of the measured and calculated dependencies can be noted. However, the calculated curves are somewhat stretched along the frequency axis. Trying to find the reason for the discrepancy the authors have established, that mast insulators influence the results due to capacitances between their caps. According to a rough guess of the insulator capacitance (Behmann and Schäfer [10]), the value of approximately $40 \mathrm{pF}$ pro capacitor could be considered. A sidearm of each mast consists of an armature and two to three insulators. Moreover, the armature parts staying under voltage build a 
capacitance to the ground. Thus, the total sidearm capacitance can be guessed to be $120 \mathrm{pF}$ per mast. The capacitance of the overhead catenary without taking into account sidearm capacitances was calculated as $10,4 \mathrm{nF} / \mathrm{km}$. With 15 to 25 masts pro kilometre, the additional capacitance due to the mast sidearms makes up 15 to $25 \%$ of the total capacitance of the catenary and therefore can not be neglected in the network model. The results obtained with the improved model show much better agreement with the measurements (curve 3 in figure 5).
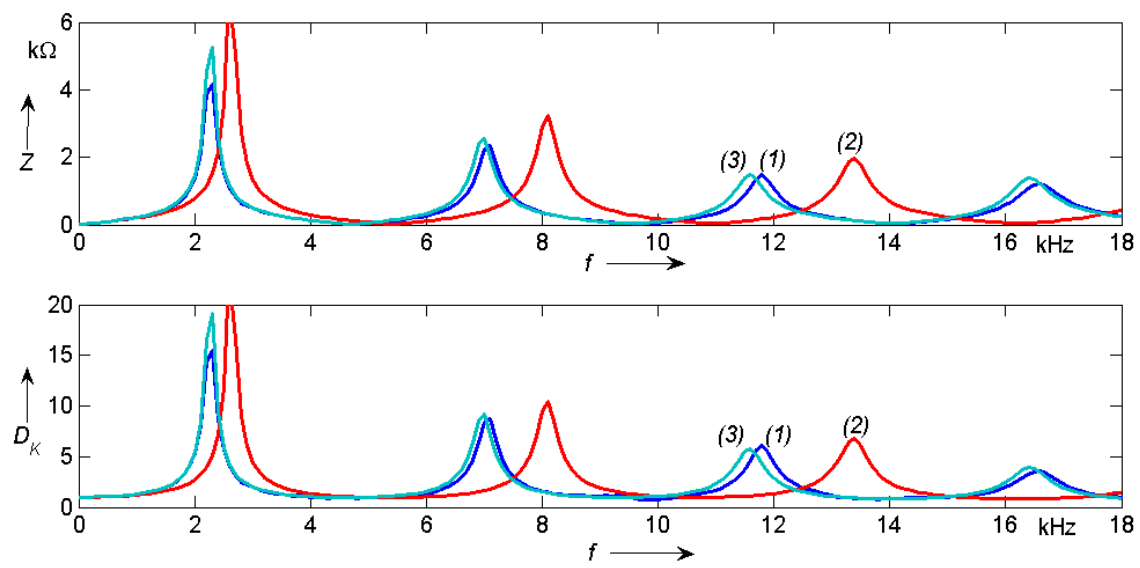

Figure 5: Calculated and measured catenary impedance and current damping ratio. (1) - measurements, (2) - calculations before measuring neglecting sidearm capacitances, (3) - calculations with the improved model taking into account sidearm capacitances.

\section{Conclusions}

In the presented new multipolar calculation method numeric errors of the common multipolar method have been reduced. This allows network computations for long, non-homogeneous traction networks for high frequencies. The method can be used to assess the maximal expected harmonic current emitted by a frequency converter of a vehicle or a station. Obtained results can be used for checking the electromagnetic compatibility of frequency converters and track circuits. Good agreement between calculation and measurement results testifies the correctness of the calculation method.

\section{References}

[1] Etxeberria-Otadui, I., Bacha, S., Bachry, A., Styczynski, Z., Fracchia, M. \& Stuart, M., Power electronic converters for railway infrastructure upgrading: advantages and potential risks. Proc. of the 2nd Int. Conf. on Critical Infrastructures. Grenoble (France), October 25-27, 2004. 
[2] ESCARV, Electrical System Compatibility for Advanced Rail Vehicles. ESCARV Final Synthesis Report, BRITE EURAM III - Industrial and Materials Technology, 2001.

[3] Xie, J., Zynovchenko, A., Jank, S. \& Klier, F., Calculation of high frequency harmonics in catenary networks. eb - Elektrische Bahnen, (103) 2005, H. 6, pp. 286-290.

[4] Zynovchenko, A., Xie, J., Jank, S. \& Klier, F., Resonance phenomena and propagation of frequency converter harmonics in the catenary of railways with single-phase A.C. Proc. of EPE'2005 $11^{\text {th }}$ European Conf. on Power Electronics and Applications. Dresden (Germany), September 2005.

[5] Putz, R., Über Streckenwiderstände und Gleisströme bei Einphasenbahnen. eb - Elektrische Bahnen, 20 (1944), H., pp.74-92.

[6] Behrends, D., Brodkorb, A. \& Hofmann, G., Calculation of catenary impedance values. eb - Elektrische Bahnen, 92 (1994), vol.4, pp. 117-122.

[7] Kontcha, A., Multipolar method for calculations in multi-conductor contact line systems for railways with single-phase A.C. eb-Elektrische Bahnen, 94 (1996), vol.4, pp. 97-102.

[8] Method for calculation of electric currents and voltages of high frequencies in catenary networks of electric railways, German patent Nr. 102005006336.5, 2005.

[9] Golub, G. H., \& van Loan, C. F., Matrix Computations, The Johns Hopkins University Press; 3-rd edition, 1996.

[10] Behmann, U. \& Schäfer, A., Effects from catenary admittance on results of short-circuit tests. eb - Elektrische Bahnen (92) 1994, H. 9, S. 260-266. 